\title{
La educación universitaria en El Salvador Un espejo roto en los 80's
}

\author{
Dr. Victor Manuel Valle \\ Con autorización de CINAS Centro de Investigación \\ y Acción Social El Salvador, C.A.
}

\section{Introducción}

Durante el decenio de los 1980 's, la sociedad salvadorena experimentó cambios notables y de gran significado. La guena civil -el factor dominante en la sociedad- afectó la totalidad de la vida económica, política y cultural.

Obviamente las universidades estuvieron en el ojo de la tormenta y su personal, sus estudiantes, sus estructuras y sus recursos fueron sometidos a fuertes tensiones. La educación superior - entendida esta primordialmente por el conjunto de universidades- se vió afectada.

El interés de este trabajo se centra alrededor del fenómeno universitario salvadoreho, principalmente en el decenio de los 1980's; asi que al hablar de educación superior se habla esencialmente de educación universitaria; por razones históricas y si se quiere, afectivas para el autor, educación universitaria es sinónimo, más o menos, de Universidad de El Salvador.

¿Por qué se escoge el decenio de los 1980's?

Los decenios, como se sabe, son caprichos cronológicos inventados por los seres humanos; pero de algo sirven para describir y analizar períodos históricos. De todas maneras, si el decenio de los 1980's comenzó en 1980 y concluyó en 1989, en ambos de esos anos se cuenta con eventos históricos de profundas implicaciones y que afectan al bl- 
nomio interactuante Universidad-Sociedad.

En 1980, a principios, una organización político militar -EI Ejército Revolucionario del Pueblo- atacó con morteros el Cuartel General de la Guardia Nacional en la capital del pais, marcado con esto un hito inaugural en la guerra civil; en marzo, el Arzobispo Oscar Arnulfo Romero fue asesinado mientras celebraba misa en una iglesia al servicio de enfermos desahuciados; en junio, la Junta de Gobierno lidereada por José Napoleón Duarte ordenó la ocupación militar del Campus principal de la Universidad de El Salvador (ocupación que duro hasta 1984); en octubre del Rector de la Universidad de El Salvador - Ing. Féliz Antonio Ulloa - murió por heridas de bala causadas por cuerpos paramiltares. Asi comenzó el decenio.

En 1989 -casi al final- sucedieron dos hechos que condensan la intensidad de la tragedia salvadorena: en noviembre, el Frente Farabundo Marti para la Liberación Nacional lanzó una ofensiva general que anodadó a analistas políticos y militares y, durante dicha ofensiva, una Unidad Militar de la Fuerza Armada Gubernamental asesinó a seis sacerdotes Jesuitas -entre ellos al Rector de la Universidad Centroamericana "José Simeón Cañas", Dr. Ignacio Ellacuria- y a dos empleadas domésticas de estos sacerdotes. $Y$ así como se inició el decenio, asl se cerró: con sociedad en guerra y universidades entrelazándose en sus más dramáticas y dolorosas circunstancias y consecuencias.

Las universidades salvadorenas, individuales y en conjunto, constituyen un micro-cosmos político; en gran medida son un reflejo de la sociedad en general, no tanto porque todas las clases sociales están representadas en ellas, sino por el hecho de que, en sus aulas, en sus auditorios $y$ en sus foros, se debaten a diario problemas que afectan a todo el pals, princlpalmente dentro del área político-social. Y ese espejo de la sociedad es el que los enfrentamientos del poder público con las universidades han roto.

Algunas lecclones -como legado- pueden extraerse de este proceso de ruptura por el que atravesaron las universidades salvadoreflas en el decenio de los 1980's. $Y$ esas lecciones pueden orientar algunas acciones necesarias para cuando el país se estabilice política y socialmente.

El propósito de este trabajo es doble: (a) describir brevemente el contexto salvadoreno, la historia singular de la universidad de EI Salvador, el surgimiento del las otras universidades y los hechos relevantes de las universidades en el decenio de los 1980's y (b) extraer conclusiones y presentar perspectivas para las universidades salvadorenas 
en el deseado proceso de la pacificación, la democratización y la reconstrucción de la sociedad salvadorena.

El carácter del trabajo - reflexiones para generar debate y elaborar hipótesis- hace que las citas al pie sean inexistentes. De todas maneras, lo escrito acá se basa en hechos de dominio público y en eventos históricos adecuadamente fundamentados.

Para contribuir a la tranquilidad de los académicos, una bibliografia básica aparece al final. porciones cnsiderables del trabajo se basan en tetimonios directos del autor.

\section{Una historla sIngular: la Unlversidad de El Salvador}

\subsection{Los origenes como parte del Estado}

En 1841, El Salvador fue el último pais en abandonar la Federacion de Centroamérica, entidad que ganó la independencia de España en 1821.

Es importante realzar que la educación universitaria en El Salvador comenzó al mismo tiempo que comenzó el Estado salvadoreno. En efecto, el cuerpo legislativo y constitucional -la Asamblea constituyente del Estado de El Salvador- que creó El Salvador como nación independiente en 1841 estableció ese mismo ano -febrero de 1841, o sea hace 150 anos - la universidad de El Salvador; quiere decir que si un poder legislativo y constituyente que creó el Estado, creó la Universidad de El Salvador, entonces el poder legislativo que disuelva el Estado de El Salvador es el único que puede disolver la Universidad de El Salvador. Por eso el grito de lucha "la universidad de El Salvador se niega a morir", que probablemente acuńó el Rector Félix Antonio Ulloa poco antes de fallecer, tiene un asidero histórico y jurídico; la Universidda de El Salvador se niega a morir, porque no puede morir sin que muera el Estado Salvadoreno.

Al fundarse la universidad de El Salvador en $1841-y$ con ello la educación universitaria en El Salvador- el Jefe de Estado era Don Juan Nepomuceno Lindo, estadista de origen hondureno; el Comandante General de las Armas era el general Francisco Malespin; la creencia común es que este General Malespin - famoso por su autoritarismo y espiritu netamente militar - fue quien ejerció una fuerte presión en el Jefe de Estado para que se diera el decreto de fundación de la Unlversidad de El Salvador.

A diferencia de las otras dos universidades más antiguas de Centro América -la de San Carlos en Guatemala y la de León en Nicara- 
gua- que eran Reales y Pontificias por haberse fundados durante el régimen colonial bajo el amparo de edictos reales y bulas papales, la Universidad de El Salvador se fundó como arte de un estado, como universidad estatal.

\subsection{Clento velntlcuatro anos de exclusividad}

Entre 1841 y 1965 - es decir por 124 anos de vida independienteen El Salvador solamente hubo una universidad; en ese período decir universidad de El Salvador era decir La Universidad, era decir educación superior; universidad pequena, obviamente, formadora de élites intelectuales y funcionarios públicos de alto nivel; ejercia funciones de docencia, investigación - a veces- y extensión cultural; al mismo tiempo servia de espacio para generar críticas a las injusticias del poder público y para alentar debates sobre problemas nacionales; los estudiantes -como es usual-constituian núcleos activos y radicales que ejercian fuerte agitación civica y política.

Para que se tenga una idea de las dimensiones de la Universidad de El Salvador, se consigna que en 1953 habia 1,016 estudiantes universitarios y en 1962 eran 2,906. Es fácil imaginarse que antes de esas épocas, el estudiante universitario salvadoreno era un conjunto de unos pocos centenares de personas. Los graduados de la secundaria bachilleres en la terminologla educacional de entonces que estudiaban principalmente con vistas a ingresar a la Universidad- eran 508 en 1957 y 1,116 en 1962.

A fines del decenio de los 1950's habla un promedio de un graduado universitario a la semana (58 en 1958); el Rector y los Decanos no eran funcionarios de tiempo completo, prácticamente no existian docentes de tiempo completo y la investigación era muy exigua.

Con todo y lo reducido de las cifras, la Universidad de El Salvador ejercia presencia importante en la vida política nacional como espacio propicio para el debate de ideas de avanzada y para formar los funcionarios civiles de los gobiernos.

\subsection{Atropellos y pérdldas sufrldas}

$Y$ ha sido ese rol de hacer crítica a las injusticias sociales y a los excesos del poder lo que le han valido a la Universidad de EI Salvador el sufrimiento de varios atropellos, siendo los historicamente más recientes: el General Hernández Martínez (1931-1944) suprimió la autonomia, fusiló estudiantes y persiguió universitarios; el Teniente Coronel Lemus (1956-1960) asaltó la Rectoria, paleó al Rector y a otras autori- 
dades universitarias, mató universitarios y reprimió reuniones públicas; el Coronel Molina (1972-1977) ocupó militarmente la Universidad prácticamente por todo un periodo presidencial; durante la presidencia del Coronel Molina ocumió la conocida masacre estudiantil del $\mathbf{3 0}$ de julio de 1975; la junta de Gobierno asaltó el campus principal de la Universidad de El Salvador en 1980; esta ocupación duró hasta 1984, período en el cual la Universidad se negó a morir y funcionó, aunque con muchas dificultades, en instalaciones alquiladas o prestadas por diversas instituciones.

Para colmo de males, en la historia reciente de la Universidad de EI Salvador se incluye terremotos e incendios. En 1955 un incendio destruyó totalmente el edificio principal de la Universidad, que estaba ubicado en el lugar que actualmente se conoce como el predio Universitario, situado frente a la Catedral Metropolitana en eterna construcción desde el incendio que la destruyó en 1951 y lugar donde está enterrado el Arzobispo Mártir Monsenor Romero. En 1965 y 1986 hubo sendos terremotos que afectaron sesiblemente el patrimonio universitario.

\subsection{Los medlos de expresión de la Unlversidad}

Una expresión de presencia universitaria en la vida nacional, como fuente de orientaciones civicas, debate político y crítica a las injusticias, fueron dos publicaciones que pretendieron ser semanales, pero que la escasez de recursos y la represión les dieron un carácter de publicaciones esporádicasde trayectoria errática.

Opinión estudiantil, ("Organo de Combate de los Estudiantes Universitarios Salvadorehos") fue fundado en' 1918 y ha aparecido desde entonces para fustigar las injusticias sociales y los excesos y abusos de los poderes constituidos. En 1956, Guillermo Manuel Ungo -dirigente político progresista recientemente fallecido- fue redactor principal de Opinión Estudiantil.

En 1963, bajo la Rectoria de Fabio Castillo Figueroa, se fundo el Semanario "El Universitario" como órgano oficial de la Universidad de El Salvador.

Estos dos medios -y otros más ocasionales - han servido para que la Universidad de El Salvador tenga presencia en la vida política y cívica de El Salvador; y para que algunos sectores conservadores la critiquen por alejarse de las funciones puramente academicas; es a través de esos medios que la Universidad de El Salvador pudo reflejar la vida política nacional, 0 al menos una muestra de ese universo polítlco. 
Con una orientación más académica y literaria que política y clvica, la Universidad de EI Salvador ha mantenido publicaciones tales como "La Universidad" que ha tenido una trayectoria igualmente ocasional, aunque la intención era mantenerla continua y periódicamente desde sus inicios el siglo pasado (Circa 1876), y "Vida Universitaria", órgano de divulgación literaria y cultural que existió en el decenio de los 1960's.

\subsection{Los fundamentos de la democracla Interna}

En 1950 hubo una huelga universitaria que desembocó en la renuncia del Rector Llerena; en ese ano se promulgó una Constitución Política (La famosa Constitución Política de 1950) de corte liberal -on un esplritu de posguerra mundial segunda modernizante, reformista, pronorteamericano y anticomunista - que reconocia la propiedad privada en función social y concedia explicitamente la automonla a la Universidad de El Salvador.

Con base en la Constitución Política de 1950 se promulgaron en 1951 la Ley Orgánica y el Estatuto Orgánico, que daban instrumentos adecuados a la Universidad de El Salvador para modernizarse tener mayor presencia en la vida pública; con estos fundamentos jurldicos y legales casi inalterados la Universidad de El Salvador funcionó con solidez y continuidad hasta 1972, ano en que el Presidente Coronel Arturo Armando Molina la ocupó militarmente.

Fue a partir de dichos instrumentos jurídicos que la Universidad de el Salvador pudo ejercitar, al interior de la institución, modelo de democracia y de participación estudiantil relevante; los estudiantes tenlan un tercio de los votos en el máximo organismo elector, treinta por ciento del poder de voto (7/24) en el Consejo Superior Universitario y veinte por ciento de dicho poder (1/5) en las Juntas Directivas de las Facultades.

Todos los órganos de gobierno académico se constituian, en los diversos electorados (profesores, estudiantes y asociaciones de graduados), por medio de elecciones directas y con voto secreto. Las asociaciones estudiantiles en todas las Facultades y la general (Asociación General de Estudiantes Salvadorehos -AGEUS-) elegian sus directivos y sus representantes en los órganos de gobierno universitario con votos directos y secretos al culminar campanas electorales que movilizanban grupos considerables de estudiantes.

Así, la Universidad de El Salvador vivió y practicó una democracia interna plena; en esto no era un reflejo de la realidad circundante, don- 
de las elecciones fraudulentas para presidentes, alcaldes y diputados eran la nota imperante y continua; realmente, con esa práctica democrática, la Universidad de El Salvador era más bien un reflejo de un sueno y de una utopla que vivia permanentemente el pueblo salvadoreno en sus anhelos de participación y de vida democrática; y al reflejar estos suenos y hacerlos realidad en su interior, la Universidad de EI Salvador era un mal ejemplo, era un espejo mal visto por los grupos gobemantes y excluyentes de la participación democrática.

\subsection{Las varlas corrlentes de pensamlento}

Para que el juego democrático interno fuera posible en la Universidad de El Salvador existlan, al menos, tres corrientes fundamentales de pensamiento que proponlan consignas, candidatos y programas de gobierno estudiantil o universitario en general. Estas corrientes eran, en el decenio de los 1960's, la izquierda revolucionaria, asociada con las posiciones marxistas o cercanas al maxismo y que se nucleaba alrededor de la Federación Estudiantil Universitaria Revolucionaria (FEUR); el socialismo democrático que, como su nombre lo indica, estaba vinculado con movimientos reformista y social demócratas y se agnupaba en la Federación Socialista Democrática (FSD); el social cristiano organizado en la Federación Revolucionaria Universitaria Social Cristiana (FRUSC) que aglutinaba a sectores afines al Partido Demócrata Cristiano (fundado en noviembre de 1960) y a grupos conservadores que haclan parte del régimen político imperante.

Para ilustración de cómo se daban estos agnupamientos en relación a la vida nacional debe saberse que, en 1966-1967, hubo una campana presidencial durante la cual la izquierda, actuando sin fisuras, presentó la candidatura del Dr. Fabio Castillo Figueroa, quien renunció a la Rectorla de la Universidad de El Salvador seis meses antes de concluir su mandato, para ser candidado a la presidencia de la República. (El Dr. Fabio Castillo durante los 1980's fue en su condición de dirigente del Partido Revolucionario de los Trabajadores Centroamericanos, miembro de la Comisión Polltico-diplomática del Frente Farabundo Martl para la Liberación Nacional); el Partido Demócrata Cristiano, que era oposición no antagónica al régimen y que ya aspiraba a ser alternativa de gobierno frente al oficialismo tradicional, sobre todo con los favores de un sector modernizante de la empresa privada y del gobierno norteamericano, presentó la candidatura del Dr. Abraham Rodriguez, quien se ha mantenido vigente en política como miembro del Partido Demócrata Cristiano y en sus relaciones profesionales y de negocios con empresas transnacionales; el Partido de Conciliación Nacional -partido oficial que contaba con el apoyo, también sin fisuras slgnl- 
ficativas, de los militares, de la oligarquia y sus allegados y del gobierno norteamericano, lazó al entonces Coronel Fidel Sánchez Hemández, quien resultó Presidente entre 1967 y 1972. Lo interesante es que, en esta coyuntura, la FEUR (que incluia entre sus dirigentes a Amtonio Handal y a Salvador Mocada) y la FSD (con sus lideres Héctor Oquell, Nelson Segovia y Enrique Escobar Barrera) apoyaron en la política nacional a Fabio Castillo y en la política universitaria una sola candidatura a Rector de la Universidad de El Salvador, el Dr. Angel Góchez Marín; los social cristianos agrupados en la FRUSC y lidereados por Rubén Zamora apoyaron, en escala nacional al demócrata cristiano Abraham Rodríguez para Presidente y para la Rector de la Universidad de El Salvador al Ing. León Enrique Cuéllar, un empresario de la instrucción vinculado con la empresa privada quien habia llegado a ser Decano de la Facultad de Ingenieria y Arquitectura de la Universidad de El Salvador con el apoyo de la izquierda. El partido oficial no tenla, al interior de la Universidad, referente organizado.

\subsection{Las reformas unlversitarlas Intentadas}

En la Universidad de El Salvador siempre hubo debate sobre las reformas necesarias para poner la universidad al servicio de mejoramiento de la sociedad. Sobre todo en este siglo, a partir de 1918 que hubo en la Universidad de Córdova, Argentina, un movimiento rerformista de repercusiones continentales, la Universidad de EI Salvador intentó varias veces impulsar movimientos de reforma.

Con frecuencia, la Universidad de El Salvador se abocaba a reformas académicas que permitieran modernizarla y ponerla al servicio efectivo de la resolución de los problemas nacionales. A fines del decenio de los 1940's por ejemplo, el Recto Carlos Llerena -quien por presión estudiantil renunció en 1950 - introdujo algunos cambios importantes, sobre todo en materla de reforma académica, pues dió un impulso tal a la investigación cientffica que creó el Instituto Tropical de Investigaciones Científicas, donde se realizaban trabajos de investigación y se elaboraban publicaciones sobre Ciencias Naturales y el medio tropical; este Instituto funcionó en un edlficio construido en la Ciudad Universitaria, la cual se comenzó a construir bajo la Rectoria del Dr. Llerena.

Cuando en 1950 los estudiantes pidieron la renuncia del Rector Llerena y proclamaron plateamientos de reformas, hablaban de participació estudiantil en el gobierno universitario, de extensión universitaria, de vinculación de los programas universitarios con los problemas nacionales, de libertad de cátedra, de autonomia universitaria; en 
suma, la llamada reforma universitaria de 1950, levantaba las banderas reformistas dentro del espiritu del movimiento de Reforma Universitaria de Córdoba, Argentina, de 32 anos antes.

En 1962, se gestó un nuevo movimiento de reforma universitaria que, inspirado e instrumentado por la izquierda, llevó en marzo de 1963 a la Rectorla de la Universidad de El Salvador al Dr. Fabio Castillo Figueroa y a los Decanatos de las Facultades a varios profesionales progresistas.

Este movimiento reformista buscaba, sobre la base de una concertación de fuerzas modernizar la universidad y ponerla al servicio directo de la solución de los grandes problemas nacionales. Eran estos los tiempos del entusiasmo del Mercado Común Centroamericano, de la Alianza para el Progreso Kennedista (aún cuando la campana para Rector y Decanos coincidió con la Crisis de los Cohetes de Cuba) y la apertura política del Presidente Coronel Julio Rivera, que abrió las válvulas políticas, para aliviar presión, por medio de la representación proporcional en la Asamblea Nacional Legislativa, que permitia a los partidos políticos de oposición, tolerada e inscrita -que exclulda obviamente a la izquierda - tener diputados nacionales de acuerdo a la proporción de votos obtenidos.

El proceso de reforma universitaria iniciado en 1963 por el Rector Fabio Castillo, fue continuado con altibajos hasta 1972 con los rectores que lo sucedieron: Rafael A.Vazquez, Angel Góchez Marín, José Marla Méndez, Gonzalo Yánez Díaz y Rafael Menjivar. Entre septiembre de 1966 y julio de 1972 - cuando la intervención militar de parte del Presidente Molina cercenó el proceso de reforma- o sea en menos de 6 anos, la Universidad de El Salvador tuvo cinco rectores.

La reforma universitaria iniciada en 1963, a la altura de 1968 ya mostraba tangibles, como puede verse en el cuadro a continuación.

Clifras comparatlvas sobre la unlversidad de El Salvador

\begin{tabular}{|lrc|}
\hline & Año 1963 & Año 1968 \\
\hline Estudiantes & 3,200 & 6,500 \\
Docentes de tiempo completo & 60 & 350 \\
Metros cuadrados de construcción & & \\
en el campus principal & 20,000 & 60,000 \\
Becarios internos & 0 & 400 \\
Centros regionales & 0 & 2 \\
Docentes, investigadores y administradores & 0 & 80 \\
Becados en el exterior & 0 & 290 (1987) \\
Graduados & 90 & \\
\hline
\end{tabular}


Estas cifras parecen insignificantes; pero para las dimensione del pals y dentro de los avances relativos muestran un proceso de reformas académica pujante y con frutos tangibles.

3. Bases para el surgimlento de las otras universidades en medio del avance de la reforma unlversitarla de la unlversidad de EI Salvador

\subsection{Coyuntura para la fundaclón de la Unlversidad Catolice}

A los productos tangibles de la reforma universitaria se agregaba la percepción - entre sectores pollticos e intelectuales- de que en la Universidad de El Salvador se estaba consolidando una actitud colectiva de compromiso con las transformaciones sociales. Habla signos y sintomas de eso.

Los domingos, se organizaban trabajos voluntarios con los que el Rector y algunos Decanos, junto a estudiantes, obreros y público en general, haclan trabajo de mantenimiento y de construcciones en la Ciudad Universitaria; los sectores conservadores dieron en llamar a esos ejercicios domingos rojos.

En el plano de bienestar estudiantil, la reforma universitaria organizo un programa de becas, residencias y comedores para estudiantes de bajos recursos económicos; con esto la composición social del estudiantado se ampliaba a sectores más populares, aunque fuera levemente.

En lo académico se crearon Departamentos de Ciencias básicas como Biología, Flsica, Matemáticas; se fundó la Facultad de Ciencias Agronómicas (un pais agrícola como El Salvador a la altura de 1963 no tenia Facultad de Ciencias Agronómicas; funcionaba una Escuela de Ingeniería Agronómica adscrita a la Facultad de Ingeniería y Arquitectura de la Universidad de El Salvador); se fortaleció la ensenanza y la investigación en las Ciencias Sociales, sobre todo en Sociologla. Y todo esto era simultáneo al hecho de que el Rector de la Universidad de El Salvador -Fabio Castillo- tenía imagen política nacional. La Universidad, para la percepción de los conservadores, era de izquierda, funcionaba bien y tenia un rector más interesado en lo político que en lo académico.

En 1964 hubo agitación en la Universidad de El Salvador; a ralz de un viaje del Rector a la Unión Soviética, donde suscribió un convenio de cooperación interuniversitaria, la derecha organizó una campana publicitaria para destituir al Rector $y$, al menos, alertar a los ciudadanos sobre el peligro comunista en la Universidad de El Salvador. 
En el plano nacional, ya el país tenía una Asamblea Legislativa pluripartidista; la oposición tolerada tenía casi la mitad de diputados en la Asamblea Nacional; el Partido Demócrata Cristiano tenia el mayor número de diputados oposicionistas.

A ralz del peligro comunista, la Federación de Padre de Familia de Colegios Católicos, movilizó corrientes de opinión para quitarle el monopolio de la educación universitaria a la universidad de El Salvador.

En ese contexto, la Asamblea Nacional Legislativa aprobó en marzo de 1965 la Ley de Universidades Privadas. Inmediatamente después, en septiembre, se aprobaron los Estatutos de la Universidad Centroamericana "José Simeón Cañas", a ser conducida por la Compania de Jesús. Entonces, la Universidad Católica, como se le llamó popularmente en sus inicios, surgió como antitesis de la universidad subversiva. La acción de reforma de la universidad de EI Salvador trajo la reacción de la fundación de la Universidad Católica.

Con el paso del tiempo, sobre todo en el marco de los mandatos del Concilio Vaticano II, de las orientaciones de la conferencia Episcopal Latinoamericana desde Medellin y de otros instrumentos teóricos y metodológicos de la llamada Teología de la Liberación, la Universidad Católica -la UCA en su diminutivo- también sería acusada de subversiva y en 1989 miembros del Ejército Nacional asesinarón a su Rector y a otros sacerdotes académicos.

\subsection{El surgimlento de las otras unlversidades}

Según la Ley de Universidades privadas, los Estatutos de las universidades se aprueban por Acuerdo del Poder Ejecutivo en el Ramo de Educación. Durante 12 anos, la Universidad Católica -cuyos Estatutos fueron aprobados en septiembre de 1965- permaneció como la única universidad privada legalmente constituida. Eran los tiempos en que para la sociedad salvadorena solamente existian dos alternativas universitarias: la Nacional y la Católica.

En 1977 se aprobaron los estatutos de otras dos universidades, la Albert Einstein -cuyos fundadores eran profesionales independlentes de las disciplinas de las ingenierias y de la ciencias exactas- y la José Matlas Delgado, que contó desde sus inicios con el apoyo de los séctores conservadores vinculados con a empresa privada. En marzo de 1979, se aprobaron los Estatutos de la Universidad Politécnlca do El Salvador. O sea que entre 1965 y 1979 se fundaron 4 universidados privadas en EI Salvador. 


\subsection{La lucha de la Unlversidad de El Salvador por preservar su Identldad}

En el decenio de los 1970's hubo hechos políticos nacionales que afectaron el desarrollo de la educación universitaria de EI Salvador. Entre 1972 y 1979 la Universidad de El Salvador vivió un periodo de mucha agitación que incluyó la intervención militar de 1972, la masacre estudiantil de 1975 en las calles de San Salvador; el cierre de 6 meses a partir de noviembre de 1976 decretado por las autoridades universitarias impuestas por el Gobierno de El Salvador la reapertura de la Universidad de El Salvador en mayo de 1977, con mecanismos represivos internos auspiciados por las autoridades impuestas; el asesinato del Rector conservador Carlos Alfaro Castillo, en septiembre de 1976; el establecimiento de un Consejo de Administración Provisional de la Universidad de El Salvador (CAPUES), investido de poderes plenos y que organizó mecanismos de mayor represión interna; el asesinato del Dr. Carlos Alberto Rodríguez, en 1978, Decano de la Facultad de Ciencias Económicas, candidato a Rector disidente del CAPUES y, por fin un Decreto Legislativo de diciembre de 1978 - cuando el Gobiemo de mano fuerte del General Carlos H. Romero era presionado principalmente por el gobierno norteamericano de Carter para abrir válvulas y liberar presiones sociales y políticas - que creaba el Consejo Directivo Provisional (CDP), formado con base en consultas con la comunidad universitaria, que organizarla elecciones directas para entregar el 5 de febrero de 1979 el poder y la conducción universitaria a autoridades legitimamente elegidas por la comunidad universitaria; el Rector elegido, Eduardo Badla Serra, renunció en junio de 1979; hubo un rector interino - Luis Argueta Antillón- de la Universidad de El Salvador, entre junio y noviembre de 1979 cuando en nuevas eleciones resultó elegido Rector el Ing. Féllx A. Ulloa.

El periodo de agitación mencionado (1972-1979) presenta varios hechos de repercusiones nacionales; la masacre estudiantil del 30 de julio de 1975, durante la cual murieron dirigentes estudiantiles de la Universidad de El Salvador (algunos fueron capturados y desaparecieron para siempre); hubo, tambiên, masacres de campesinos que causaron conmoción; sucedió, además, el anuncio de la pretendida Transformación nacional, ofrecida en 1976 por el gobierno del Coronel Molina, que se convirtió en el Reculón nacional cuando dicho presidente, ante presiones de los sectores conservadores, desmanteló un programa de reformas sociales y económicas. En el mismo perlodo ocurrió la transición del presidente Coronel Molina al Presidente Coronel Romero (1977), quienes llegaron a la Presidencia por medio de ostensibles fraudes electorales. 
Esta agitación nacional y las rupturas de la organización de la Universidad de El Salvador tuvieron sus repercusiones en el reordenamiento de la educación universitaria. Los estudiantes necesitaban nuevas instituciones para optar a una carrera universitaria y obtener un tifulo académico; los docentes de la Universidad de el Salvador buscaba formas de estabilizar su ocupación académica. $Y$ asi ayudó a crear las condiciones para el surgimiento de las otras universidades.

El comienzo de este periodo de tensiones (1972-1979) lo marcó la varias veces mencionada intervención militar de la Universidad de EI Salvador, el 19 de julio de 1972. Inmediatamente después de esta intervención, grupos de estudiantes y docentes de la Universidad de El Salvador volvieron sus ojos a la Universidad Católica, la única alternativa de institución universitaria en esos momentos. Ante la Universidad de El Salvador intervenida, el vació de la crítica y la protesta era llenado paultinamente por la Universidad Católica; y al tornarse la UCA creciente contestaria, eran necesarias otras universidades más identificadas con el régimen. $Y$ asi se creo, por ejemplo, en diciembre de 1977, la Universidad José Matias Delgado, cuando era Presidente el General Carlos H. Romero, conocido por su conducta represiva y su afinidad con los sectores empresariales más conservadores.

El espejo universitario comenzaba a fracturarse; el espacio universitario salvadorefo comenzaba a tomarse un mosaico. Era entonces, válido referirse a las universidades con calificativos distintos. Para las opiniones simplistas. La universidad de El Salvador era foco de subversión, la UCA expresaba la opinión de la Iglesia Católica y a lo mejor del Partido Demócrata Cristiano; la José Matías Delgado representaba el ideario de la empresa privada y por tanto tenia afinidades con el gobiemo central, el ejército y su partido oficial el Partido de Conciliación Nacional. Tales era los estereotipos que se manejaban.

\section{El decenlo de los 1980's: El espejo roto}

\subsection{El preámbulo de los 1980 's}

En la antesala de los 1980's en 1979- hubo mucha agitación en el pals. Varias masacres con motivaciones politicas sacudieron a la sociedad; muy conocida fue la masacre de la Catedral Metriopolitana, en mayo de 1979, filmada por un periodista dada a conocer en el mundo corno expresión condensada de la conmoción que habia en el Salvador. En julio de 1979, el dictador Somoza caía en Nicaragua ante el empuje de una insurrección popular lidereada por los Sandinlstas; Carter, como Presidente de los Estados Unidos, alentaba una politica 
de derechos humanos que buscaba mejorar la conducta de los gobernantes pro-norteamericanos de la región que tenlan reputación represiva. En esa coyuntura sucedió, el 15 de octubre de 1979, el derrocamiento de Presidente derechista General Romero, sentándose con esto las bases para el comienzo de un nuevo decenio.

\subsection{Los casos extremos}

El Salvador tuvo, entre 1980 y 1989, un perlodo de gran agitación social y política. El sector universitario -liel reflejo de las realidades y de las utopias del pals - también se conmovió.

En enero de 1980, Guillermo Ungo lidereó una renuncia masiva de elementos progresistas - muchos de ellos de raices universitarias- del gobierno instalado en octubre de 1979 , debido a que grupos observadores de militares y civiles, comprometidos con el régimen anterior, haclan negatorias todas las medidas de cambio que prentendlan los reformistas en el gobierno. El 2 de enero de 1980, la más grande concentración popular en la historia del pais, fue dispersada a balazos por francotiradores apostados en azoteas de edíficios públicos en el centro de la capital. En marzo de 1980, la Junta de Gobierno -ahora bajo la cara civil del IIder Demócrata Cristiano José Napoléon Duarto- emprendió un proceso militarlzado de reforma agraria concebido en los marcos conceptuales y operaclonales de asesores norteamericanos (e.g. Roy Prosterman) con experiencia en reformas agrarias contralnsurgentes en el Sur Este aslátlco; las reformas eran, en todo caso, para secar el agua al pez o dicho de otra forma para sacar del agua al pez, siendo el agua el sector campesino ávido de tierra para trabajarla y el pez el movimiento guerrlllero que levantaba la bandera de la revolución social.

Monsenor Oscar Arnulio Romero se refirió a estas medidas como reformas con represión o reformas tenidas de sangre. El 24 de marzo de 1980, Monsenor Romero fue asesinado; en junio de 1980 el campus principal de la Universidad de El Salvador -en San Salvador- fue ocupado militarmente después de una icurslón violenta de las fuerzas militares del Gobierno de El Salvador (esta ocupación duró hasta 1984); en octubre de 1980 el Rector de la Universidad de EI Salvador murió de heridas de bala causadas por elementos paramilitares.

$Y$ con esos hechos se inauguró el decenio que hubo de presenciar una dispersión y una atomización de la educación universitaria en EI Salvador; decenio que concluiria con el asesinato -en noviembre de 1989- del Rector, el Vice-Rector y el Director del Instituto de Investigaciones Sociales de la Universidad Católica, los sacerdotes jesuitas 
Ignacio Ellacuria, Ignacio Martín-Baró y Segundo Montes respectivamente, (otros tres sacerdotes y dos empleadas domésticas de la UCA murieron en esa matanza perpetrada por unidades del ejército salvadoreno - según ya es el dominio público- durante la ofensiva del FMLN de noviembre de 1989).

Hay cierta similitud en ambos hechos de muerte de rectores. EI Rector Ulloa comenzó su actividad político-universitaria, como directivo estudiantil de la Sociedad de Estudiantes de Ingenieria Salvadorenos en 1963, representando sectores moderados de izquierda democrática dentro de la Universidad de EI Salvador; cuando se graduó fue, sobre todo, un técnico y un docente interesado en resolver los problemas nacionales dentro de su área profesional de la Ingeniería Industrial y esa posición de compromiso creciente lo llevó a la Rectorla de la Universidad de El Salvador como un acadérnico comprometido en serio con el cambio social.

La Universidad Católica surgió como una entidad universitaria conservadora; poco a poco - a través de su involucramiento en el análisis de la realidad nacional - los académicos religiosos de la UCA fueron tomando posiciones de mayor compromiso. El Rector Ignacio Ellacuría puso su pensamiento al servicio del esclarecimiento de la problemática social y polltica.

O sea que la muerte de ambos rectores (Ulloa y Ellacuria) ilustra un hecho aterrador: cuando los universitarios de El Salvador llevan las funciones clásicas de la universidad (docencia, investigación y extensión o acción social) hasta sus últimas consecuencias en favor de las mayorias y del cambio social a fondo, el régimen los castiga con la muerte.

\subsection{La guerra Interna y la dispersión de las universidades}

Este trabajo no es un análisis de la guerra interna que ha determinado toda la vida social, política, económica y cultural de EI Salvador desde 1980. Sobre eso hay abundantes referencias. Lo que se anota en este trabajo es que la educación universitaria en EI Salvador ha experimentado, durante el decenio la agudización de la guerra interna en El Salvador, una enorme atomización y dispersión. El espejo del sector universitario se ha roto.

Ante los ataques mortales a los dirigentes universitarios, frente a las intervenciones militares de las instalaciones universitarias (principalmente de la Universidad de El Salvador) y con atentados dinamlteros a edificios de la Universidad de El Salvador y de la Universidad Católlca, 
un fenómeno ha signado el desarrollo universitario en El Salvador en los anos 1980's: la proliferación de las universidades privadas.

Al amparo de la legislación que permite el establecimiento de las universidades privadas, entre 1980 - ano en el que comenzó la actual guerra civil y los campuses de la Universidad de El Salvador $\infty$ menzaron a sufrir un periodo de 4 anos de ocupación militar de parte del Gobierno de El Salvador- y 1989, según información contenida en documentos oficiales, el Ministerio de Educación autorizó los Estatutos de 30 universidades privadas.

Al finalizar el decenio de los 1980's (1989, según la Mernoria de Labores 1989-1990 del Ministerio de Educación de la República de EI Salvador), habla una matricula universitaria total de 75,000 estudiantes aproximadamente; el $40 \%$ de estos estudiantes estaban en la Universidad de El Salvador; un 9\% estaban en la Universidad Católica; quiere decir que la otra mitad de la matrícula universitaria (37,500 estudiantes) estaba dispersa en unas 30 universidades privadas, algunas de ellas minúsculas instituciones con nombres tales como "Modular Abierta", "Cristiana de las Asambleas de Dios", y "del Vendedor Salvadorenos". (Ver cuadro anexo).

La guerra contribuyó al fraccionamiento de la educación universitaria. Los cierres y asedlos de la Universidad de El Salvador —principal institución universltarla del pals en términos cuantitativos- generaron vacios; las personas callilcadas para seguir estudios universitarias y obtener dlplomas, necesitaban nuevas opciones para matricularse; los docentes sin empleo, necesitaban dónde colocar su fuerza de trabajo intelectual. $Y$ asi, olertas y demandas fueron encontrando sus cauces y niveles y se generó la educación universitaria fraccionada que se tiene actualmente en El Salvador, como uno de los legados del decenio de los 1980's.

\subsection{Consecuenclas del frecclonamlento}

\section{¿A qué lleva este fracclonamiento unlversitarlo?}

En to político, hay una doble dimensión como efecto del fraccionamiento del sector universitario. La demanda por educación universitaria y la presión social para preservar la Integridad de las universidades más contestatarias (e.g. Universidad de El Salvador y Universidad Católica) son mejor manipuladas por el régimen y por su expresión concreta del gobiemo de turno, si hay instituciones alternativas donde seguir estudios universitarios. Por otro lado, en medio de una variedad y abundancia (relativamente) de universidades, los poderes constituidos 
del régimen pueden actuar como instancia manipuladora de recursos públicos y componedora de conflictos. Los fondos públicos, aunque escasos, pueden canalizarse - por medio de diversas formas - hacia las instituciones mejor portadas con el régimen.

En lo cultural, podria anotarse que, sobre todo en el decenio de los 1980 's, ha aparecido, además de la universidad jesuita de los 1960's, las universidades con signo religioso: Evangélica, Salesiana, Cristiana de las Asambleas de Dios, Luterana. Es inferible que algunas de las otras, sin ser religiosas también tiendan a representar una suerte de credo político o social; así, cada universidad pasaria a ser -en virtud del fraccionamiento - una tribuna o trinchera para determinado credo y la idea de universidad para crear, preservar y desarrollar manifestaciones de cultura nacional quedaria disminuida.

En lo académico, el fraccionamiento universitario, tal como se ha dado en El Salvador, es fuente de potencial mediocridad. Es casi imposible que una institución nueva y pequena, sin recursos, sin filosoffa educacional, dedicada a ofrecer una o dos carreras, pueda cumplir con propiedad y calidad las funciones esenciales de la universidad en docencia, investigación y extensión o acción social.

La universidad debe ser un centro superior donde se realice trabajo académico intelectual para elaborar, críticamente, conocimientos nuevos, donde se formen los profesionales que tendrán a cargo la organización y conducción de los programas estratégicos para el desarrollo nacional; donde se fomente la preservación y divulgación del patrimonio cultural y artistico de la nación; donde, en fin, se articulen las relaciones de la academia con los procesos de transformación económica, social y política del país.

La dispersión universitaria en El Salvador, tal como se dio en el decenio de los 1980's, hace difícil el cumplimiento de la misión de la universidad, en los términos arriba apuntado.

\section{Concluslones y perspectlvas: a restaurar el espejo}

La tesis central de este trabajo es que la educación universitaria de EI Salvador -como espejo de la realidad y de las utopias de la sociedad salvadorena - fue fracturada en el decenio de la agudización de la guerra interna (1980-1989). Algunas conclusiones y propuestas de acción pueden esbozarse con base en los capitulos precedentes.

\subsection{Conclusiones}

1. La Universidad de El Salvador está histórica y jurídicamente 
entrelazada con la esencia del Estado salvadoreno; el mismo poder legislativo y constitucional creo ambas instancias -Estado y universidad-, casi simultáneamente, en 1841.

2. El Salvador es un pals empobrecido y sufrido, pequefio y subdesarrollado, que demanda una educación universitaria modema y vigorosa que ayude al desarrollo nacional en beneficio de las mayorlas.

3. La Universidad de El Salvador tiene una historia de reformas académicas relevantes, de práctica de democracia interna y de ejercicio de pluralismo en el sector progresista de las fuerzas sociales y políticas del pais.

4. Las universidades privadas surgieron - a partir de 1965- como contrapeso de la Universidad de El Salvador; desde 1972 y más agudamente desde 1980, las unlversidades privadas han surgido para llenar vacios generados por los cierres y asedios sufridos por la Universidad de El Salvador.

5. La guerra interna - factor determinantes de toda la vida nacionaprovocó una dispersión y una atomización de la educación universitaria de EI Salvador.

6. La Universidad de El Salvador, al concluir el decenio de los 1980's, continuaba como la principal institución universitaria -en términos cuantitativos- del pals, con el $\mathbf{4 0 \%}$ de la matricula total.

7. La existencia de muchas unlversidades en términos relativosparece ser un fenómeno irreversible en El Salvador; muchas universidades, sobre todo las surgldas antes de 1980 , se han institucionalizado. (A fines de 1989, las 5 universidades fundadas antes de 1980 - Universidad de El Salvador, Universidad Católica, Universidad Albert Einstein, Universldad José Matias Delgado y Universidad Politécnica- contaban con el $5 \%$ de la matrícula total de 75,000 estudiantes).

8. La dispersión del sector universitario en El Salvador ha posibilitado por lo menos, tres hechos:

a) El manipuleo político de parte de los poderes constituidos del régimen para actuar como instancia componedora entre las varias instituciones de educación superior.

b) La desnaturalización de la idea clásica de universidad al servicio de una cultura nacional, al generar universidades privadas con signos políticos, religiosos y sociales predeterminados. 
c):-El deterioro acadérnico universitario, al aceptar socialmente instituciones que no tienen los medios para cumplir adecuadamente las funciones esenciales de la universidad, cuales son la docencia, la investigación y la extensión o acción social. (Un dato patético se registra en la p. 20 de un documento del Ministerio de Educación de El Salvador -Educación en cifras 1988-1989- cuando se informa que en 1986 la Universidad del Vendedor Salvadoreho tuvo veinticuatro (sic) estudiantes y a partir de 1987 no se consigna matricula alguna).

\subsection{Perspectlvas y sugerenclas}

Las conclusiones enunciadas llevan a imaginar perspectivas y a sugerir futuros cursos de acción. El espejo roto debe restaurarse, 0 al menos los varios espejos existentes deben ordenarse y amnonizarse para que, en su conjunto, reflejen adecuadamente la realidad nacional; pero que, además de reflejarla, actúen sobre ella iluminando y generando energlas necesarias para el desarrollo nacional. A la metáfora mencionada debe llegarse con medidas concretas y operacionales como las que se mencionan a continuación:

\subsubsection{Coordlnación entre todas las unlversidades}

Las universidades -de común acuerdo y sobre bases de decisión voluntaria- deben constituir un Consejo Nacional de Rectores que se reúna periódicamente y que funja como instancia coordinadora de políticas generales.

En este Consejo Nacional de Rectores las universidades tendrlan un voto ponderado, de acuerdo a ciertos indicadores que emanen de las estadísticas oficiales, como por ejemplo matricula total, número de profesores a tiempo completo, investigaciones realizadas. La determinación de estos indicadores debería ser uno de los primeros actos autónomos del Consejo.

EI Consejo deberá tener una oficina ejecutora, la cual debe ser financiada con aportes del Gobierno Central, un porcentaje del presupuesto de cada universidad que voluntariamente acepte participar y fondos atternativos obtenidos en fuentes financieras de desarrollo (Bancos, fundaciones y Agencias para el desarrollo internacional pertenecientes a paises desarrollados).

Las atribuciones mínimas de esta oficina ejecutora serian: a) recoger y divulgar informaciones universitarias; b) compilar y compartir, en lo posible, elaboraciones metodológicas de trabajo académlco (planeamiento, administración, desarrollo curricular, organización de Investl- 
gaciones) para uso de las universidades; c) asesorar a las universidades que lo soliciten en la forma de elaborar perfiles de proyectos, susceptibles de ser financiados, como donativo o como préstamo, por fuentes financieras externas; d) coordinar capacitación y perfeccionamiento de personal universitario en la aplicación de metodologlas de trabajo universitario y e) opinar, con fundamentos académicos, sobre la pertinencia de aprobar el establecimiento o cierre de una entidad universitaria.

\subsubsection{Fortaleclmlento académlco pertinente}

Las universidades salvadorenas deben fortalecer áreas de trabajo académico que le dan razón de ser a las universidades, como por ejemplo los estudios de posgrado, el desarrollo de la investigación científica, el desarrollo de las ciencias básicas. El actual énfasis en docencia para otorgar títulos de pre-grado, debe ser cambiado.

En cuanto a áreas de apoyo al trabajo académico, es necesario crear mecanismos de coordinación interinstitucional, instancias de Acreditación de carreras, facultades y universidades, formas seguras de financiamiento, sistemas de información gerencial, programas de carrera académica y de desarrollo integral del personal universitario y medios de relacionamiento internacional.

Para ponerse al frente de las tareas de la reconstrucción -las cuales se esperan que ocurran en los 1990's como consecuencia inmediata de una paz que resulte de la solución negociada del conflicto armado- las universidades deberán tener propuestas claras sobre sus funciones clásicas, sus metodologlas de trabajo, su identidad, su interacción con la sociedad y su manejo de temas novedosos como la conservación del medio ambiente y el manejo de los recursos naturales, la resolución de confllctos y la búsqueda de consensos, la relación creativa con el sector productivo de bienes y servicios, la interacción con entidades de nuevo tipo en la sociedad civil (e.g. Las Organizaciones no Gubernamentales que expresan una forma nueva y dinámica de participación de la sociedad organizada).

\subsubsection{La restauración de la Unlversidad de El Salvador}

La Universidad de El Salvador, la Primada de las universidades salvadorenas y el Alma Máter de la educación universitaria del país - cuyo lema Hacia la Libertad por la cultura denota su vocación libertaria, su proyección social y su carácter de entidad de cultura nacional- debe ser restaurada con el aporte de sus integrantes. 
En el momento histórico que se habla de negociación para democratizar y de consenso para reconstruir es necesario que la Universidad de El Salvador sea un modelo de pluralismo para la reconstrucción y una avanzada de un eventual y necesario modelo nacional de consenso.

La dinámica histórica, la composición social de los estudiantes y de los docentes y los enfrentamientos políticos en el pais han configurado, en la Universidad de El Salvador, una comunidad universitaria con peso específico hacia la izquierda del espectro político nacional. $Y$ este es un hecho que, por de pronto, está vigente y parece irreversible en el periodo histórico inmediato.

La misma dinámica política de El Salvador ha dado a la izquierda salvadorena un espacio relevante en la política nacional. La aceptación del Gobierno de El Salvador de negociar, desde abril de 1990, con el auspicio de la Secretaría General de la Organización de las Naciones Unidas, con la insurgencia expresada en el FMLN y los recientes resultados electorales de marzo de 1991 que dan a la izquierda -aún con el masivo abstencionismo y las acusaciones de fraude oficial- una porción significativa de miembros de la Asamblea Nacional Legislativa, son muestras de esta afirmación.

El desafío de la comunidad universitaria de la Universidad de EI Salvador consiste en lograr cómo, reconociendo su carácter progresista, puede emprender caminos de consensos y pluralismo que restauren la calidad académica de la Universidad, la modernicen y la reformen y la pongan al servicio de la reconstrucción del pais y de la afirmación de una cultura nacional sin exclusiones.

Independientemente de los acontecimientos recientes, en la Universidad de El Salvador, por los que han tomado posesión nuevas autoridades legítimamente constituidas, es pertinente hacer un planteamiento de sugerencias a ser consideradas autónomamente por la comunidad de la Universidad de El Salvador.

1. Las fuerzas fundamentales de la comunidad universitaria de la Universidad de El Salvador deben encontrar una personalidad de consenso, preferiblemente independiente, de excelencia académica y de trayectoria respetable para proponerlo a los organismos pertinentes como candidato a Rector. En consulta con esta personalidad deberán encontrarse los candidatos para los otros puestos de las autoridades centrales de elección.

2. Los intelectuales y los políticos del sector progresista de EI Salvador que, como se ha dicho en este trabajo, tiene ubicación en la 
Universidad de El Salvador, deben elaborar y suscribir una plataforma mínima de consenso para que las autoridades legítimamente constituidas la reciban como una propuesta para iniciar y consolidar, en el marco de un consenso nacional, la restauración académica de la Universidad de El Salvador, para hacer de dicha Universidad el Centro Superior por excelencia de la cultura nacional. El Rector, con la ayuda del equipo central, sería el responsable de impulsar la plataforma de consenso.

3. La comunidad universitaria y las fuerzas progresistas organizadas deberán suscribir, como complemento a la plataforma mínima, una especie de pacto social interno, por medio del cual se comprometerán a que en el periodo en que se ponga en práctica la plataforma de consenso, no habrá agltaciones con fines políticos partidarios.

4. Inmediatamente después de instaladas las autoridades centrales, se iniciará al interior de la Unlversidad de El Salvador, un proceso de libre juego de ideas y debates constructivos para que, en el marco de una democracia particlpatlva interna y sin exclusiones ideológicas de ningún tipo, se elijan nuevos Decanos y Vice-decanos de Facultad.

5. El cuerpo de autorldades ya completo declararia su apoyo al tomento de todas las lormas de expresión y organización intelectual al interior de la Unlversldad de El Salvador, ofrecería un programa claro y blen organzado de restauración académica al servicio del camblo soclal, expllcltarla medidas efectivas de bienestar estudiantil y demandarla del Goblerno de la República un compromiso serio y Ilrme de respetar la autonomla de la Universidad de El Salvador. 
Anexo

Unlversldades prlvadas de El Salvador

y ano de aprobaclón de sus estatutos

\begin{tabular}{|ll|}
\hline \multicolumn{1}{|c|}{ Universidades } & Añ \\
\hline 1. Centroamericana "José Simeón Canas" & 1965 \\
2. Albert Einstein & 1977 \\
3. José Matias Delgado & 1977 \\
4. Politécnica de El Salvador & 1979 \\
5. Alberto Masferrer & 1980 \\
6. Tecnológica & 1981 \\
7. Evangelica & 1981 \\
8. Técnica Latinoamericana & 1981 \\
9. Leonardo Da Vinci & 1981 \\
10. Occidental & 1981 \\
11. Francisco Gavidia & 1981 \\
12. Nueva San Salvador & 1981 \\
13. Las Américas & 1982 \\
14. Autonoma de Santa Ana & 1982 \\
15. Americana & 1982 \\
16. Gerardo Barrios & 1982 \\
17. Salvadorena & 1982 \\
18. Católica de Occidente & 1982 \\
19. De Oriente & 1982 \\
20. Santaneca de Ciencia y Tecnologla & 1982 \\
21. Salvadorena Isaac Newton & 1982 \\
22. De Sonsonate & 1982 \\
23. Moldular Abierta & 1982 \\
24. Pedagógica de El Salvador & 1982 \\
25. Salvadorena "Berry" & 1983 \\
26. Cristiana de las Asambleas de Dios & 1983 \\
27. Don Bosco & 1984 \\
28. Del Vendedor Salvadoreno & 1984 \\
29. Educación Integral (sistema modular) & 1984 \\
30. Thomas Alva Edison & 1985 \\
31. De La Paz & 1987 \\
32. Metropolitana de El Salvador & 1987 \\
33. De Administración de Negocios & 1988 \\
34. Luterana & 1988 \\
\hline
\end{tabular}

Cuadro elaborado con base en las Memorias Anuales de Labores del Miniotorlo de Educación, República de El Salvador. 


\section{BIBUOGRAFIA}

Del Mazo, Gabriel, Estudiantes y Gobierno Universitario (Segunda Edición). Argentina: Editorial "El Ateneo", 1955.

Del Mazo, Gabriel Reforma Universitaria y Cultura Nacional Argentina: Editorial Raigal, 1955.

El Salvador. Constitucion Politica 1983.

El Salvador. Ley de Universidades Privadas. Decretos Legislativos 244 (24 de marzo de 1965) y 250 (26 de enero de 1966).

Inter-American Development Bank. Economic and social progress in Latin America 1989 Report Washington D.C.: Inter-American Development Bank, 1989.

López-Vallecillos, Italo. El Periodismo en El Salvador El Salvador: Editorial Universitaria, 1964.

Merlos, Salvador Ricardo. "El Constitucionalismo Centroamericano en la Mitad del Siglo XIX", La Universidad Nos. 3-4 1960, El Salvador, pp. 9-53.

Ministerio de Educación. Diagnóstico de la Situación de la Educación Universitaria en El Salvador. El Salvador, 1989.

Ministerio de Educación. Educación Superior en Cifras 1988-1989. El Salvador: Dirección General de Educación Superior, Ministerio de Educación 1990.

Ministerio de Educación. Memoria de Labores 1989-1900 El Salvador, 1990.

Pérez Brignoli, Héctor Breve Historia de Centroamérica. (Tercora Edición), España: Alianza Editorial, 1988, (Primera Edición, 1985).

The World Bank. Demographic Issues and Prospects (A World Bank Country Study on El Salvador). Washington d.C., 1979.

The Word Bank. World Development report 1989. USA: Oxford University Press/The World bank, 1989.

Universidad Centroamericana "José Simeón Cañas". La Investigación y la docencia en la Educación Universitaria de El Salvador. Estudio realizado por la UCA en el marco de un proyecto PREDE-OEA. versión mimeografiada, abril de 1990.

Universidad de El Salvador. "Datos Históricos", Gula-1962. El Salvador: Editorial Universitaria, 1962.

Valle, Victor Manuel El Estudiante en la Reforma Universitaria. El Salvador: Facultad de Humanidades. Universidad de El Salvador, 1969.

Valle, Victor Manuel. El Salvador en los 1960's Testimonio y documentos. (EI despertar de una conciencia Popular Organizada). versión Mimeografiada en proceso de publicación para 1991.

Valle, Victor Manuel. "La Evaluación en los Organizaciones Universitarias", Anuario-1986 Unión de Universidades de América Latina (UDUAL), México D.F., pp. 269-282.

Valle, Victor Manuel. "Proposiciones sobre Autonomia Universitaria", Universidades-101. Unión de Universidades de América Latina (UDUAL). México d.F. Julio-septiembre de 1985 pp. 131-146.

Valle, Victor Manuel. "Transformaciones docentes en la Reforma Universitaria de El Salvador". Revista Educación. Nos. 8-9, abril-septiembre de 1966, 
Universidad de El Salvador.

Vu My World Population Projections - 1985. USA: The World Bank and the Johns Hopkins University Press. 1985. 